\title{
Nivel de ecoeficiencia en energía y residuos sólidos en los estudiantes de la Facultad de Ingeniería de la Universidad Nacional Jorge Basadre Grohmann, Tacna
}

Level of eco-efficiency in energy and solid waste in the students of the Faculty of Engineering of the Jorge Basadre Grohmann National University, Tacna

${ }^{1, a}$ Hennrry Brady Huisa Cutipa

${ }^{2, \mathrm{~b}}$ Carlos Armando Polo Bravo

ARTÍCULO ORIGINAL
${ }^{1}$ Gerencia de Operaciones, EXSA S.A
Tacna, Perú. E-mail: hhuisac@gmail.com
${ }^{2}$ Universidad Nacional Jorge Basadre
Grohmann. Departamento Académico de
Física. Tacna, Perú. E-mail:
polodomando@gmail.com

a ORCID: 0000-0002-4233-8929

'ORCID: 0000-0001-9491-2323

Palabras clave: Conciencia ambiental, ecoeficiencia, estudiantes, valores.

Keywords: Environmental awareness, ecoefficiency, students, values.

Información adicional

Presentado: 20/09/2020

Aprobado: 28/12/2020

\section{RESUMEN}

La investigación tiene como objetivo determinar el nivel de ecoeficiencia en energía y residuos sólidos de los estudiantes de la Facultad de Ingeniería de la Universidad Nacional Jorge Basadre Grohmann, considerando la conciencia ambiental y valores. La muestra para el estudio fue de 286 estudiantes de las cinco escuelas profesionales de la facultad, considerando un error del $5 \%$. Se aplicó el método de muestreo no probabilístico, técnica por cuotas estratificando la población en sectores e instrumentos de interés. La confiabilidad de los instrumentos se determinó mediante el coeficiente del Alpha de Cronbach, cuyos valores fueron: $0.850,0.944$ y 0.740 ; obteniendo una alta confiabilidad de los instrumentos. Se determinó que los estudiantes tienen una relación de ecoeficiencia en energía positiva muy débil (5.9\%), en tanto que en residuos sólidos una relación positiva media $(26.3 \%)$; y niveles, regular de conciencia ambiental $(53.5 \%)$ y alto en valores (67.9\%). En la prueba de hipótesis se evidenció que existe una relación positiva débil entre las variables.

ABSTRACT
The objective of the research is to determine the level of eco-efficiency in
energy and solid waste of the students of the Faculty of Engineering of the
Jorge Basadre Grohmann National University, considering
environmental awareness and values. The sample for the study was 286
students from the five professional schools of the faculty, considering an
error of 5\%. The method of non-probabilistic sampling was applied, a
technique by quotas, stratifying the population in sectors and instruments
of interest. The reliability of the instruments was determined by means of
Cronbach's Alpha coefficient, whose values were: 0.850 , 0.944 and
0.740; obtaining a high reliability of the instruments. It was determined
that the students have a very weak positive energy ecoefficiency ratio
(5.9\%), while in solid waste a medium positive ratio (26.3\%); and regular
environmental awareness levels (53.5\%) and high values (67.9\%). In the
hypothesis test it was shown that there is a weak positive relationship
between the variables.




\section{INTRODUCCIÓN}

El contexto universitario es propicio para la transmisión de información técnica para el cuidado del ambiente. Por una parte, habría que considerar si la preocupación sobre las propiedades del entorno ambiental se halla en relación a una conducta adecuada hacia la protección del mismo (Chero et al., 2019). Ante ello, Dehaene (2015) refiere que el cuidado del ambiente involucra percepciones, pensamientos, sentimientos y sociabilidad. Asimismo, los estudios acerca del comportamiento ambiental despiertan un interés creciente; cada vez que la degradación ambiental acelerada se atribuye, en parte, a deficiencias en este tipo de comportamiento (Oskamp, 2000; Zelezny \& Schultz, 2000). Por otro lado, los estudios que se dedican a la explicación del comportamiento ambiental generalmente prueban relaciones directas entre diversas variables identificadas, tales como los valores (Corraliza \& Martín, 2000; García \& Real, 2001). Los estudios enfocados en adquirir información sobre las actitudes a favor de la conservación del ambiente se han abordado desde la psicología ambiental. De hecho, estos estudios evalúan las actitudes con el objetivo de obtener un conocimiento acerca de cuanto ha avanzado la conciencia ambiental de las personas sobre la preservación del ambiente(Gil et al., 2017).

Una de las alternativas de solución para mitigar y/o eliminar estos problemas ambientales es fortaleciendo la conciencia ambiental en los estudiantes universitarios, quienes actúan como vectores humanos (Vargas et al., 2010). Asimismo, Gomera (2008) afirma que la conciencia ambiental son conocimientos, percepciones, conductas y actitudes con dimensiones que, en conjunto, conforman el concepto de conciencia ambiental. Es decir, entender cómo influyen las acciones de cada día en el ambiente y cómo esto afecta el futuro de nuestro espacio. Del mismo modo, De la Llata et al. (2019) afirman que los valores son elementos constitutivos de todo proceso de educación, cambio y desarrollo social. Los valores son cualidades estructurales que se adhieren en las personas, instituciones y sociedades, influyendo en los comportamientos ambientales de manera directa o indirecta, ya sea por la sensibilización de los individuos a conjuntos particulares de consecuencias de las condiciones ambientales (Dietz et al., 2002). Actualmente, la educación universitaria suele estar marcada por la transmisión de valores conservacionistas, algunos transmitidos mediante la inclusión de los ejes transversales y otros por las asignaturas relativas al ambiente (Cayón \& Pernalete, 2011). Además, Ros \& Gouveia (2001) afirman que los valores sirven para establecer relaciones entre las prioridades valorativas y los comportamientos. De hecho, Bolzan (2008) afirma que conocer cómo estos se organizan y se articulan puede ofrecer pistas del modo en que se produce un comportamiento ambiental. La honestidad es un valor y uno de los componentes más importantes de una personalidad saludable (Briceño, 2011). El despertar a la conciencia ecológica es la aprehensión real y profunda de actitudes conscientes en beneficio propio, de los otros y del planeta (Cabana, 2017).

A lo largo de los años, la ecoeficiencia ha tenido un amplio rango de interpretaciones, todas tienen en común el uso eficiente de los recursos naturales (Cabana, 2017). De la misma forma, el prefijo "eco" hace referencia a los asuntos ambientales, tanto a lo ecológico como a lo económico. La ecoeficiencia es una herramienta que relaciona outputs e inputs de manera tal que mientras menores sean los insumos utilizados, más eficiente será el proceso. La ecoeficiencia emerge como una respuesta administrativa frente a los asuntos asociados con los desechos provenientes de los procesos productivos (Núñez, 2006). La Universidad debería tomar medidas de ecoeficiencia orientadas al cuidado y equilibrio del ambiente a través del uso racional de los recursos, ahorro de energía, agua y gestión sostenible de residuos sólidos, entre otros aspectos (Montesinos, 2011). Entre las funciones de las universidades se encuentra la formación y capacitación de sus egresados, buscando la modernización conforme a la realidad, es decir, promoviendo la investigación continua y sostenible en temas de desarrollo industrial, y reaprovechamiento de los recursos para lograr el menor impacto ecológico posible. 
Nivel de ecoeficiencia en energía y residuos sólidos en los estudiantes de la Facultad de Ingeniería de la Universidad Nacional Jorge Basadre Grohmann, Tacna

Las políticas para desarrollar la desmaterialización de la economía se basan en incrementar la eficiencia energética, especialmente en los procesos productivos en todos los sectores económicos (De Felipe, 2010). Se están proponiendo políticas internacionales para el uso de la energía, de tal forma que se busquen alternativas que generen energía sin contaminar, como la energía eólica, que es limpia y proporciona las necesidades energéticas necesarias. Sin energía el mundo se paraliza, ya que con ella se cubren todas las necesidades de la población (Cabana, 2017). Por otro lado, Ortiz et al. (2010) afirman que los residuos sólidos comprenden todos los desechos que provienen de actividades humanas, y normalmente son sólidos.

En efecto, la determinación de los factores del consumo nos ayuda a ver cuáles son las preferencias de los estudiantes, los cambios que podrían tener a futuro y los estímulos que se podrían aplicar para adelantar nuevas políticas y que los consumidores individuales se vean influenciados por distintos criterios a la hora de decidir por algún producto o servicio (Cabana, 2017).

En la Facultad de Ingeniería (FAIN) de la Universidad Nacional Jorge Basadre Grohmann (Tacna, Perú) se pudo observar diferentes tipos de residuos sólidos tanto en las aulas como en las áreas verdes. Asimismo, se puede señalar que los estudiantes de la facultad no tienen hábitos de apagar los interruptores de las luces o equipos electrónicos que se utilizan; al mismo tiempo, no se aprovecha la luz solar. En tal sentido, esto constituye un problema importante que debe de resolverse a través de estrategias de formación de competencias en los futuros profesionales y de políticas de sostenibilidad ambiental que ayuden a superar estas deficiencias. En tal sentido, se realiza esta investigación con el objetivo de determinar la relación de la conciencia ambiental y los valores en el nivel de ecoeficiencia en los estudiantes de la FAIN de la Universidad Nacional Jorge Basadre Grohmann (UNJBG).

\section{MATERIAL Y MÉTODOS}

La investigación se llevó a cabo en la FAIN, con una población estudiantil de 1126 estudiantes matriculados en el 2019. Mediante la ecuación de población finita, se obtuvo una muestra de 286 estudiantes, distribuyéndose por cada escuela profesional de la siguiente manera: $13.64 \%$ de ingeniería química, 25.17 $\%$ de ingeniería informática y sistemas, $18.88 \%$ de ingeniería mecánica, $20.28 \%$ de ingeniería de minas y $22.03 \%$ de ingeniería metalúrgica.

Se diseñó el cuestionario considerando las variables de la conciencia ambiental en base al cuestionario de Vosmediano y San Juan (2015) con 37 ítems escala tipo Likert, conformado por las siguientes dimensiones: cognitiva (10 ítems), afectiva (16 ítems), conativa (5 ítems) y activa (6 ítems); valores en base al cuestionario de Casas (2015) con 40 ítems escala tipo Likert conformado por las siguientes dimensiones: honestidad (20 ítems) y despertar a la conciencia ecológica (20 ítems); y nivel de ecoeficiencia en energía y residuos sólidos en base al cuestionario de García \& Marrou (2014) con 31 ítems escala tipo Likert, conformado por las siguientes dimensiones: energía (13 ítems) y residuos sólidos (18 ítems). La validez del cuestionario se realizó mediante el juicio de expertos y su confiabilidad a través del coeficiente Alpha de Cronbach, mediante una encuesta realizada a 31 estudiantes. El cuestionario se aplicó a estudiantes del segundo, sexto y décimo ciclo de estudios entre los meses de octubre y noviembre del 2019, con un tiempo de duración de 15 a 20 minutos; la aplicación del cuestionario fue anónima y de participación voluntaria.

El objetivo específico fue determinar la relación y/o grado de asociación de la conciencia ambiental y los valores en la ecoeficiencia de los estudiantes de la FAIN. Los resultados obtenidos del cuestionario fueron agrupados. Los datos analizados no siguen una distribución normal, por lo tanto, se realizó una prueba no paramétrica de variables ordinales, prueba de Rho de Spearman. Los análisis se realizaron con el paquete estadístico SPSS v18 con un nivel de significancia de 0.05 . 
Tabla 1

Escalas, valores, nivel y rango de variables

\begin{tabular}{llc}
\hline \multicolumn{1}{c}{ Variable } & \multicolumn{1}{c}{ Escalas y valores } & Niveles y rangos \\
\hline & 1: Muy poco & \\
2: Poco & Alto $(136-185)$ \\
Conciencia ambiental & Medio & Rastante \\
$(37$ items $)$ & 5: Mucho & Bajo $(37-85)$ \\
& 1: Nunca & \\
& 2: Casi nunca & \\
3: A veces & Alto $(147-200)$ \\
Valores & 4: Casi siempre & Regular $(93-146)$ \\
$(40$ items $)$ & 5: Siempre & Bajo $(40-92)$ \\
& 1: Sí & \\
\hline Ecoeficiencia & $0:$ No & óptimo $(21-31)$ \\
$(31$ items $)$ & & Regular $(10-20)$ \\
\end{tabular}

\section{RESULTADOS}

Para la confiabilidad del cuestionario, las tres variables obtuvieron valores mayores a 7.0. Esto significa que el instrumento es adecuado para los propósitos de esta investigación. La variable valores tuvo como resultado un valor mayor a 9.0, siendo adecuado para la toma de decisiones (Tabla 2).

Tabla 2

Confiabilidad de los instrumentos

\begin{tabular}{lcc}
\hline \multicolumn{1}{c}{ Variables } & $\begin{array}{c}\text { Alfa de } \\
\text { Cronbach }\end{array}$ & $\begin{array}{c}\text { Número } \\
\text { de ítems }\end{array}$ \\
\hline Conciencia ambiental & 0.850 & 37 \\
Valores & 0.944 & 40 \\
Ecoeficiencia & 0.740 & 31 \\
\hline
\end{tabular}

En la Tabla 3, observamos que en los estudiantes de la FAIN predomina el nivel regular de conciencia ambiental (53.5\%), seguido de un nivel alto (46.2\%) y finalmente un nivel bajo $(0.3 \%)$.

Tabla 3

Nivel de la conciencia ambiental en los estudiantes de la FAIN

\begin{tabular}{lccc}
\hline \multicolumn{1}{c}{ Nivel } & Rango & Frecuencia & Porcentaje \\
\hline Bajo & $37-85$ & 1 & $0.3 \%$ \\
Regular & $86-135$ & 153 & $53.5 \%$ \\
Alto & $136-185$ & 132 & $46.2 \%$ \\
\hline Total & & 286 & $100.0 \%$ \\
\hline
\end{tabular}


En la Tabla 4, observamos que en los estudiantes de la FAIN predomina el nivel alto de valores (67.9 \%), seguido de un nivel regular $(31.1 \%)$ y finalmente un nivel bajo $(1.0 \%)$.

Tabla 4

Nivel de valores en los estudiantes de la FAIN

\begin{tabular}{|c|c|c|c|}
\hline Nivel & Rango & Frecuencia & Porcentaje \\
\hline Bajo & $40-92$ & 3 & $1.0 \%$ \\
\hline Regular & $93-146$ & 89 & $31.1 \%$ \\
\hline \multirow[t]{2}{*}{ Alto } & $147-200$ & 194 & $67.9 \%$ \\
\hline & & 286 & $100.0 \%$ \\
\hline
\end{tabular}

En la Tabla 5, observamos que en los estudiantes de la FAIN predomina el nivel regular de ecoeficiencia (72.4\%), seguido de un nivel óptimo (14.0\%) y finalmente un nivel no óptimo (13.6\%).

\section{Tabla 5}

Nivel de ecoeficiencia en los estudiantes de la FAIN

\begin{tabular}{lccc}
\hline \multicolumn{1}{c}{ Nivel } & Rango & Frecuencia & Porcentaje \\
\hline No óptimo & $0-9$ & 39 & $13.6 \%$ \\
Regular & $10-20$ & 207 & $72.4 \%$ \\
ó ptimo & $21-31$ & 40 & $14.0 \%$ \\
\hline Total & & 286 & $100.0 \%$ \\
\hline
\end{tabular}

En la Tabla 6, observamos que en los estudiantes de la FAIN predomina el nivel regular de ecoeficiencia en energía (74.8\%), seguido de un nivel no óptimo (15.8\%) y finalmente un nivel óptimo $(9.4 \%)$.

Tabla 6

Nivel de ecoeficiencia en energía en los estudiantes de la FAIN

\begin{tabular}{lccc}
\hline \multicolumn{1}{r}{ Nivel } & Rango & Frecuencia & Porcentaje \\
\hline No óptimo & $0-4$ & 45 & $15.8 \%$ \\
Regular & $5-9$ & 214 & $74.8 \%$ \\
óptimo & $10-13$ & 27 & $9.4 \%$ \\
\hline Total & & 286 & $100.0 \%$ \\
\hline
\end{tabular}

En la Tabla 7, observamos que en los estudiantes de la FAIN predomina el nivel regular de ecoeficiencia en residuos sólidos con un (54.9\%), seguido de un nivel no óptimo (30.4\%) y finalmente un nivel óptimo con $(14.7 \%)$.

\section{Tabla 7}

Nivel de ecoeficiencia en residuos sólidos en los estudiantes de la FAIN

\begin{tabular}{lccl}
\hline \multicolumn{1}{c}{ Nivel } & Rango & Frecuencia & Porcentaje \\
\hline No óptimo & $0-6$ & 87 & $30.4 \%$ \\
Regular & $7-12$ & 157 & $54.9 \%$ \\
óptimo & $13-18$ & 42 & $14.7 \%$ \\
\hline Total & & 286 & $100.0 \%$ \\
\hline
\end{tabular}


El análisis de relación de las variables conciencia ambiental y nivel de ecoeficiencia, considerando un nivel de significancia menor a 0.05 y un coeficiente Rho de Spearmann $\left(r_{s}\right)$ de 0.226 (Tabla 8), indica que existe una relación positiva débil entre ambas variables. Del mismo modo, se observa que las variables valores y nivel de ecoeficiencia, considerando un nivel de significancia menor a 0.05 y un coeficiente $R h o$ de Spearmann $\left(r_{s}\right)$ de 0.244 (Tabla 8), indican que existe una relación positiva débil entre ambas variables.

Tabla 8

Relación de la conciencia ambiental y valores con el nivel de ecoeficiencia en los estudiantes de la FAIN

\begin{tabular}{|c|c|c|c|c|}
\hline & de Spearman & $\begin{array}{c}\text { Conciencia } \\
\text { ambiental } \\
\text { (agrupado) }\end{array}$ & $\begin{array}{c}\text { Valores } \\
\text { (agrupado) }\end{array}$ & $\begin{array}{c}\text { Ecoeficiencia } \\
\text { (agrupado) }\end{array}$ \\
\hline \multirow{3}{*}{$\begin{array}{l}\text { Conciencia } \\
\text { ambiental } \\
\text { (agrupado) }\end{array}$} & Coeficiente correlación & 1.000 & 0.385 & 0.226 \\
\hline & Sig. (bilateral) & - & 0.000 & 0.000 \\
\hline & $\mathrm{N}$ & 286 & 286 & 286 \\
\hline \multirow{3}{*}{$\begin{array}{l}\text { Valores } \\
\text { (agrupado) }\end{array}$} & Coeficiente correlación & 0.385 & 1.000 & 0.244 \\
\hline & Sig. (bilateral) & 0.000 & -- & 0.000 \\
\hline & $\mathrm{N}$ & 286 & 286 & 286 \\
\hline \multirow{3}{*}{$\begin{array}{l}\text { Ecoeficiencia } \\
\text { (agrupado) }\end{array}$} & Coeficiente correlación & 0.226 & 0.244 & 1.000 \\
\hline & Sig. (bilateral) & 0.000 & 0.000 & -- \\
\hline & $\mathrm{N}$ & 286 & 286 & 286 \\
\hline
\end{tabular}

El análisis de relación de las variables conciencia ambiental y nivel de ecoeficiencia en energía, considerando un nivel de significancia mayor a 0.05 y un coeficiente Rho de Spearmann $(r$ ) de 0.059 (Tabla 9 ), indica que no existe un grado de asociación entre ambas variables. Del mismo modo, se observa que las variables valores y nivel de ecoeficiencia en energía, considerando un nivel de significancia menor 0.05 y un coeficiente Rho de Spearman $\left(r_{s}\right)$ de 0.211 (Tabla 9), indican que existe una relación positiva débil entre ambas variables.

Tabla 9

Relación de la conciencia ambiental y valores con la dimensión energía de la ecoeficiencia en los estudiantes de la FAIN

\begin{tabular}{|c|c|c|c|c|}
\hline & lo de Spearman & $\begin{array}{l}\text { Conciencia } \\
\text { Ambiental } \\
\text { (agrupado) }\end{array}$ & $\begin{array}{c}\text { Valores } \\
\text { (agrupado) }\end{array}$ & $\begin{array}{l}\text { D. Energía } \\
\text { (agrupado) }\end{array}$ \\
\hline \multirow{3}{*}{$\begin{array}{l}\text { Conciencia } \\
\text { ambiental } \\
\text { (agrupado) }\end{array}$} & Coeficiente correlación & 1.000 & 0.336 & 0.059 \\
\hline & Sig. (bilateral) & -- & 0.000 & 0.322 \\
\hline & $\mathrm{N}$ & 286 & 286 & 286 \\
\hline \multirow{3}{*}{$\begin{array}{l}\text { Valores } \\
\text { (agrupado) }\end{array}$} & Coeficiente correlación & 0.336 & 1.000 & 0.11 \\
\hline & Sig. (bilateral) & 0.000 & -- & 0.000 \\
\hline & $\mathrm{N}$ & 286 & 286 & 286 \\
\hline \multirow{3}{*}{$\begin{array}{l}\text { D. Energía } \\
\text { (agrupado) }\end{array}$} & Coeficiente correlación & 0.059 & 0.211 & 1.000 \\
\hline & Sig. (bilateral) & 0.000 & 0.000 & -- \\
\hline & $\mathrm{N}$ & 286 & 286 & 286 \\
\hline
\end{tabular}

El análisis de relación de las variables conciencia ambiental y nivel de ecoeficiencia en residuos sólidos, considerando un nivel de significancia menor a 0.05 y un coeficiente Rho de Spearmann $(r$ ) de 0.173 (Tabla 10), indica que no existe un grado de asociación entre ambas variables. Del mismo modo, se observa que las variables valores y nivel de ecoeficiencia en energía, considerando un nivel de significancia menor 0.05 y un coeficiente Rho de Spearman ( $r$ ) de 0.135 (Tabla 10), indican que existe una relación positiva débil entre ambas variables. 
Nivel de ecoeficiencia en energía y residuos sólidos en los estudiantes de la Facultad de Ingeniería de la Universidad Nacional Jorge Basadre Grohmann, Tacna

Tabla 10

Relación de la conciencia ambiental y valores con la dimensión residuos sólidos de la ecoeficiencia en los estudiantes de la FAIN

\begin{tabular}{|c|c|c|c|c|}
\hline & ho de Spearman & $\begin{array}{l}\text { Conciencia } \\
\text { Ambiental } \\
\text { (agrupado) }\end{array}$ & $\begin{array}{c}\text { Valores } \\
\text { (agrupado) }\end{array}$ & $\begin{array}{l}\text { D. Residuos } \\
\text { Sólidos } \\
\text { (agrupado) }\end{array}$ \\
\hline \multirow{3}{*}{$\begin{array}{l}\text { Conciencia } \\
\text { ambiental } \\
\text { (agrupado) }\end{array}$} & Coeficiente correlación & 1.000 & 0.336 & 0.173 \\
\hline & Sig. (bilateral) & -- & 0.000 & 0.003 \\
\hline & $\mathrm{N}$ & 286 & 286 & 286 \\
\hline \multirow{3}{*}{$\begin{array}{l}\text { Valores } \\
\text { (agrupado) }\end{array}$} & Coeficiente correlación & 0.336 & 1.000 & 0.135 \\
\hline & Sig. (bilateral) & 0.000 & -- & 0.003 \\
\hline & $\mathrm{N}$ & 286 & 286 & 286 \\
\hline \multirow{3}{*}{$\begin{array}{l}\text { D. Residuos } \\
\text { Sólidos } \\
\text { (agrupado) }\end{array}$} & Coeficiente correlación & 0.173 & 0.135 & 1.000 \\
\hline & Sig. (bilateral) & 0.003 & 0.003 & -- \\
\hline & $\mathrm{N}$ & 286 & 286 & 286 \\
\hline
\end{tabular}

\section{DISCUSIÓN}

Los estudiantes de la FAIN manifiestan una alta preocupación por la contaminación ambiental que afecta a su entorno; además muestran altos niveles de compromiso verbal sobre la necesidad de informar a la gente de la importancia y efectos de los problemas ambientales, así como de participar en actividades en favor del ambiente; sin embargo, esto no es coherente con su comportamiento real. Debe señalarse que los estudiantes reciben una formación ambiental durante su estancia universitaria, pero no se ve expresada en sus hábitos.

Los estudiantes de la FAIN tienen un nivel regular de conciencia ambiental (53.5\%), seguido de un nivel alto (46.2\%) (Tabla 3), esto se puede comparar con los estudios realizados por Coya (2001) y Toledo (2017), donde obtuvieron una puntuación regular en la muestra de los estudiantes. Igualmente, se corroboró con el estudio realizado por Miranda (2017), donde se concluyó que el nivel de conciencia ambiental en los estudiantes fue de mediano cumplimiento, describiendo a este sector de la comunidad universitaria con un nivel de conocimiento, de información y de disposición relativamente aceptables, y con valores, creencias, actitudes y comportamientos ambientales adecuados. Finalmente, Aragonés \& Amérigo (1991), en su investigación, determinaron que los estudiantes de la Universidad Complutense de Madrid muestran una conciencia ambiental positiva; de acuerdo con esta escala, los estudiantes manifestaron una muy buena actitud y afectividad, además de un claro conocimiento de la situación actual y futura del ambiente, del mismo modo, tienen un compromiso activo en la realización de actividades propias y colectivas favorables al ambiente. Estos resultados son contrarios a nuestra investigación, donde a pesar de tener conocimiento sobre la problemática ambiental actual, esto no se ve reflejado en las actitudes y comportamientos ambientales de los estudiantes de la FAIN.

Los estudiantes de la FAIN tienen un nivel alto de valores con un $67.9 \%$, seguido de un nivel regular con un $31.1 \%$ (Tabla 4), cabe indicar que los valores son muy importantes según Pato \& Tamayo (2006), quienes investigaron el papel mediador de las creencias ambientales en relación a los valores y el comportamiento proambiental activo, demostrando que los valores de universalismo, benevolencia y autodirección pueden influir en los comportamientos ambientales de manera indirecta a través de las creencias ambientales. Los autores suponen que los valores actúan activando las creencias ambientales.

Con respecto a la ecoeficiencia, el $72.4 \%$ de los estudiantes de la FAIN alcanzó un nivel regular, mientras que el $14 \%$ obtuvo un nivel óptimo (Tabla 5). Asimismo, un $74.8 \%$ alcanzó un nivel regular respecto a la dimensión energía del nivel de ecoeficiencia (Tabla 6); es conveniente recalcar que Linares (2009) manifestó que la eficiencia energética puede contribuir al logro de los objetivos ambientales y, en especial, a 
los compromisos de reducción de emisiones de gases de efecto invernadero. Al mismo tiempo, se obtuvo un $54.9 \%$ en el nivel regular respecto a la dimensión residuos sólidos del nivel de ecoeficiencia (Tabla 7) comparativamente con los estudios realizados por Herrera et al. (2016), quienes determinaron que los estudiantes universitarios (respecto al manejo de los residuos sólidos) tienen un alto nivel de favorabilidad, representado en un reporte de conductas de ahorro, reutilización del papel y manejo y clasificación de los residuos sólidos, cuyos porcentajes oscilan entre un $96.2 \%$ y $84.8 \%$. Sin embargo, la reutilización y clasificación de los residuos sólidos no se vio reflejado en los estudiantes de la FAIN a pesar de contar con un regular nivel de conciencia ambiental sumado a una deficiente política ambiental por la parte de la UNJBG.

La conciencia ambiental y el nivel de ecoeficiencia tienen una relación positiva débil del $22.6 \%\left(r_{s}=0.226\right.$ y $\left.p_{\text {value }}<0.05\right)$. Asimismo, los valores y el nivel de ecoeficiencia tienen una relación positiva débil del $24.4 \%\left(r_{s}\right.$ $=0.244 \mathrm{y} p_{\text {value }}<0.05$ ), comparado con el estudio realizado por Gil, Guerra \& Olivares (2017), donde obtuvieron que las variables que corresponden a la actitud y comportamiento relacionado a la conciencia ambiental y ecoeficiencia $\left(r_{s}=0.50\right.$ y $\left.p_{\text {value }}<0.05\right)$ mostraron una relación estadísticamente significativa, moderada y directamente proporcional entre la actitud y el comportamiento hacia el ambiente en estudiantes universitarios. Del mismo modo, Rivera y Rodríguez (2009) demostraron una relación directa débil entre actitudes y comportamientos ambientales $\left(r_{s}=0.30\right.$ y $\left.p_{\text {value }}<0.05\right)$ de forma similar a otros estudios en universitarios. Asimismo, en los resultados sobre la función de las dimensiones del nivel de ecoeficiencia, se observa que la conciencia ambiental no incide significativamente en la dimensión energía del nivel de ecoeficiencia $\left(r_{s}=0.059\right.$ y $\left.p_{\text {value }}>0.05\right)$, pero sí incide significativamente en la dimensión residuos sólidos del nivel de ecoeficiencia $\left(r_{s}=0.173\right.$ y $\left.p_{\text {value }}<0.05\right)$, indicando una relación positiva débil. Todo lo contrario sucede con la variable valores, que sí inciden significativamente con la dimensión energía del nivel de ecoeficiencia $\left(r_{s}=0.211\right.$ y $\left.p_{\text {value }}<0.05\right)$ y con la dimensión residuos sólidos del nivel de ecoeficiencia $\left(r_{s}=\right.$ 0.135 y $\left.p_{\text {value }}<0.05\right)$, indicando en ambos casos una relación positiva débil. Estos resultados coinciden con el estudio realizado por De la Llata et al. (2019), quienes demostraron inconsistencias en los encuestados sobre "qué dicen o piensan" y "lo que finalmente hacen". Sin embargo, los valores ambientales que a nivel grupal seleccionaron, en la práctica cotidiana, no se reflejan de manera suficiente al analizar sus actitudes, comportamientos y las medidas que dicen tomar. Asimismo, tal como lo señalan Thompson \& Barton (1994), quienes han encontrado un bajo grado de asociación entre actitudes proambientalistas y conductas orientadas a la conservación de los recursos.

El bajo grado de asociación entre las variables puede estar influenciada por otras de tipo motivacional, cognitivas, de creencias o perceptivas, que no han sido consideradas en esta investigación; de hecho, un adecuado comportamiento ambiental será manifestado entre los estudiantes con mayores niveles de compromiso actitudinal. Lo más importante es que los comportamientos inadecuados pueden ser modificados durante los primeros ciclos de formación universitaria, asimismo, la explicación inmediata es que dichas conductas generalmente conllevan un cierto grado de sacrificio e inconvenientes que dificultan su realización de modo que, a pesar de una aparente preocupación por el ambiente, muchos de los estudiantes no aceptan cambios en sus hábitos de vida. De igual manera, De la Llata et al. (2018) afirmaron que es necesario adoptar medidas ambientales que favorezcan la congruencia entre los valores y actitudes que se dicen tener, para verlos reflejados en acciones; sobre todo, considerando lo que destaca y advierte Cánovas (2002), quien recomienda la urgente necesidad de formar valores ambientales que busque un máximo beneficio económico y la máxima rentabilidad y productividad a corto plazo.

El estudio presenta algunas limitaciones, al aplicarse una prueba correlacional, que no expresa relaciones de causalidad entre las variables estudiadas. Los resultados únicamente señalan relaciones de asociación entre ellas y no permiten afirmar que aumentando o fomentando actitudes proambientales vayan a mejorar los comportamientos. En tal sentido, se considera que una muestra mayor hubiera permitido que los valores de las variables sigan una distribución normal para la aplicación de una prueba paramétrica. Finalmente, es conveniente recalcar que estos resultados preliminares servirían de base para estudios posteriores, que además sugieren la necesidad de mejorar los comportamientos ambientales en los estudiantes de la FAIN. 
Nivel de ecoeficiencia en energía y residuos sólidos en los estudiantes de la Facultad de Ingeniería de la Universidad Nacional Jorge Basadre Grohmann, Tacna

\section{CONCLUSIONES}

Las variables de la conciencia ambiental, valores y nivel de ecoeficiencia tienen una correlación positiva débil, indicando que las variables de la conciencia ambiental y valores inciden en el nivel de ecoeficiencia de los estudiantes de la FAIN.

Las variables de la conciencia ambiental y la dimensión energía del nivel de ecoeficiencia tienen una correlación positiva muy débil; del mismo modo, los valores y la dimensión energía del nivel de ecoeficiencia tienen una correlación positiva débil. Por lo tanto, se concluye que la conciencia ambiental no incide en la dimensión energía del nivel de ecoeficiencia, a diferencia de los valores que tienen una incidencia baja en los estudiantes de la FAIN, algo que se corrobora en los comportamientos ambientales de los estudiantes de la FAIN en sus actividades diarias dentro y fuera de la facultad.

Las variables de la conciencia ambiental y la dimensión residuos sólidos del nivel de ecoeficiencia tienen una correlación positiva media. Del mismo modo, los valores y la dimensión residuos sólidos del nivel de ecoeficiencia tienen una correlación positiva débil. Por lo tanto, se concluye que la conciencia ambiental y valores tiene una incidencia baja en la dimensión de residuos sólidos del nivel de ecoeficiencia de los estudiantes de la FAIN.

\section{REFERENCIAS}

Aragonés, J., Amérigo, M., Sevillano, V., \& Cortés, B. (2005). La estructura de las creencias sobre la problemática medioambiental. Psicothema, 17(2), 257-262.

Bolzan, C. (2008). Sistemas de Gestión ambiental y comportamiento proambiental de trabajadores fuera de la empresa: aproximación de una muestra brasileña (tesis doctoral). Universidad de Barcelona.

Briceño, A. (2011, 6 de abril). La Honestidad. Blog http://wwwwlahonestidad.blogspot.pe/

Cabana, A. (2017). Conciencia ambiental, valores y ecoeficiencia en la Gerencia de Servicios a la Ciudady Medio Ambiente Lima cercado, 2016 (tesis doctoral). Universidad César Vallejo.

Cayón, A., \& Pernalete, J. (2011). Conciencia ambiental en el sistema educativo venezolano. Revista Electrónica de Humanidades, Educación y Comunicación Social, 11, 163-186.

Chero, V., Oruna, J., Jaimes, S., \& Tovar, M. (2019). Relación entre conciencia ambiental y conducta pro ambiental en estudiantes de primer ciclo de la Universidad María Auxiliadora Lima-Perú. Ciencia \& Desarrollo, 18(24), 66-73.https://doi.org/10.33326/26176033.2019.24.787

Corraliza, J., \& Martín, R. (2000). Estilos de vida, actitudes y comportamientos ambientales. Medio Ambiente y Comportameinto Humano, 1(1), 31-56. https://doi.org/10.1590/s141381232004000400008

Coya, M. (2001). La Ambientalización de la Universidad. Un estudio sobre la formación ambiental de los estudiantes de la universidad de Santiago de Compostela y la política ambiental de la institución (tesis doctoral). Universidad de Santiago de Compostela.

De Felipe, J. (2010). Energía. Cátedra UNESCO de Sostenibilidad. Universitat Politécnica de Catalunya. http://portalsostenibilidad.upc.edu/detall_01.php

De la Llata, M., Juárez, A., \& Sampedro, M. (2019). Valores y comportamientos ambientales de estudiantes de educación media superior y superior. Revista UPIICSA Investigación Interdisciplinaria, 5(2), $33-50$.

Dehaene, S. (2015). La conciencia en el cerebro descifrando el enigma de cómo el cerebro elabora nuestros pensamientos. Siglo XXI.

Dietz, T., Kalof, L., \& Stern, P. (2002). Gender, Values, and Environmentalism. Social Science Quarterly, 83(1), 353-364. https://doi.org/10.1111/1540-6237.00088

García, R., \& Real, E. (2001). Valores, actitudes y creencias: hacia un modelo predictivo del ambientalismo. Medio Ambiente y Comportamiento Humano: Revista Internacional de Psicología Ambiental, 2(1), 21-43. 
Gil, H. M., Guerra, G. M., \& Olivares, O. A. (2017). Actitudes y Comportamientos Ambientales en estudiantes de la carrera de Licenciatura en Enfermeria y Licenciatura en Psicología. Revista Caribeña de Ciencias Sociales.

Gomera, A. (2008). La conciencia ambiental como herramienta para la educación: conclusiones y reflexiones de un estudio en el ámbito universitario. Centro Nacional de Educación Ambiental.

Herrera, K., Acuña, M., Ramírez, M., \& De la Hoz, M. (2016). Actitud y conducta proecológica de jóvenes universitarios. Opción. Revista de Ciencias Humanas y Sociales, 13, 456-477.

Jollands, N., Lermit, J., \& Patterson, M. (2004). Aggregate eco-efficiency indices for New Zealand-a principal components analysis. Journal of Environmental Management, 73, 293-305. https://doi.org/10.1016/j.jenvman.2004.07.002

Linares, P. (2009). Eficiencia energética y medio ambiente. Economía y Medio Ambiente, 847, 75-92.

Miranda, D. (2017). Medición de la conciencia ambiental en estudiantes de ciencias agrarias de la Universidad Nacional de Tumbes. Revista Humanidades e Innovación, 4(2), 108-123.

Núñez, G. (2006). El Sector Empresarial en la Sostenibilidad Ambiental: Ejes de Interacción. CEPAL.

Ortiz, O., Pasqualino, J. C., Díez, G., \& Castells, F. (2010). The environmental impact of the construction phase: An application to composite walls from a life cycle perspective. Resources, Conservation \& Recycling, 54, 832-840.https://doi.org/10.1016/j.resconrec.2010.01.002

Oskamp, S. (2000). A sustainable future for humanity? How can psychology help? American Psychologist, 55(5), 496-508. https://doi.org/10.1037/0003-066X.55.5.496

Pato, C., \& Tamayo, A. (2006). Valores, creencias ambientales y comportamiento ecológico de activismo. Medio Ambiente y Comportamiento Humano, 7(1), 51-66.

Ros, M., \& Gouveia, B. (2001). Psicología social de los valores humanos. Desarrollos teóricos, metodológicos y aplicados. Biblioteca nueva.

Toledo, P. (2017). Nivel de conocimiento y participación de los estudiantes de enfermería sobre el cuidado ambiental Universidad Privada San juan Bautista sede Chorrillos, Junio 2016 (tesis de pregrado). Universidad Privada San Juan Bautista.

Vargas, C., Vázques, L., Gutiérrez, G., Vargas, M., \& Fernández, C. (2010). Conciencia ambiental en estudiantes de Enfermería Básica. CONHISREMI, Revista Universitaria de Investigación y Diálogo Académico, 6(3), 18-28. http://conhisremi.iuttol.edu.ve/pdf/ARTI000103.pdf

Zelezny, L., \& Schultz, P. (2000). Promoting Environmentalism. Journal of Social Issues, 56(3), 365-371. https://doi.org/10.1111/0022-4537.00172 